\title{
P381: Legionella priority control: development of a comprehensive tool for healthcare settings
}

\author{
P Del Giudice ${ }^{1 *}$, L Arnoldo $^{1}$, D Goi ${ }^{1}$, R Cocconi ${ }^{2}$, B Casini ${ }^{3}$, G Gurnari $^{1}$, U Moscato ${ }^{4}$, ML Cristina $^{5}$, S Brusaferro ${ }^{1}$ \\ From 2nd International Conference on Prevention and Infection Control (ICPIC 2013) \\ Geneva, Switzerland. 25-28 June 2013
}

\section{Introduction}

Despite scientific and technological advances, Legionella continues to be a major threat to patients in healthcare settings. To be effective the control of Legionella need a competent attention based on a correct risk assessment and on consequent operative choices.

\section{Objectives}

A tool called Legionella Priority Control [LPC], was designed to be comprehensive, easily implementable and able to define intervention priorities in the healthcare setting.

\section{Methods}

LPC was developed within a project funded by the Italian Ministry of University through the following stages:

- Review of literature to identify all the possible critical points in Legionella control;

- Classification of all selected critical points in three independent areas of interest: management, water distribution system and patient susceptibility;

- Identification of several critical points for each area;

- Split of each critical point into items to be answered in form of questionnaire;

- Classification of items through a multidisciplinary consensus into 4 risk categories ( 1 - best practice/low to no risk, 2 - possible Legionella presence (not evidence based)/ medium risk, 3 - probable Legionella presence (evidence based)/high risk, 4 - demonstrated Legionella presence/ very high risk);

- Analysis of the 3 areas scores in order to define a general risk score and a structure related risk score;

- Use of a risk matrix (including a feasibility score to be assigned by local practitioners linked to the setting) to define final priorities.

\section{Results}

LPC includes:

- A check list of items which, once filled in, allows the definition of existing problems (3 areas of interest, 15 critical points and 76 items);

- A risk matrix which allows definition of an initial list of priorities;

- A subjective feasibility score which allows definition of priorities tailored to the local setting.

\section{Conclusion}

LPC offers to the infection control practitioner an easier yet comprehensive way to define priorities for Legionella control tailored to the local healthcare setting.

\section{Disclosure of interest}

None declared.

\section{Author details}

${ }^{1}$ University of Udine, Italy. ${ }^{2}$ Azienda Ospedaliero-Universitaria Santa Maria della Misericordia, Udine, Italy. ${ }^{3}$ University of Pisa, Pisa, Italy. ${ }^{4}$ Catholic University of the Sacred Heart, Rome, Italy. ${ }^{5}$ University of Genoa, Genoa, Italy.

Published: 20 June 2013

doi:10.1186/2047-2994-2-S1-P381

Cite this article as: Del Giudice et al:: P381: Legionella priority control: development of a comprehensive tool for healthcare settings. Antimicrobial Resistance and Infection Control 2013 2(Suppl 1):P381. 